\title{
Fabrication of Highly Ordered Arrays of Platinum Nanoparticles Using Direct Laser Interference Patterning
}

\begin{tabular}{|r|l|}
\hline Journal: & ACS Applied Materials \& Interfaces \\
\hline Manuscript ID: & draft \\
\hline Manuscript Type: & Letter \\
\hline Date Submitted by the \\
Author: & \\
\hline Complete List of Authors: & $\begin{array}{l}\text { Acevedo, Diego; Universidad Nacional de Rio Cuarto, CHEMISTRY } \\
\text { Salavagione, Horacio; Instituto deCiencia y Tecnnología de } \\
\text { Polímeros } \\
\text { Lasagni, Andrés; Saarland University } \\
\text { Morallon, Emilia; Universidad de Alicante, Departamento de } \\
\text { Quimica Fisica } \\
\text { Mücklich, Frank; Saarland University } \\
\text { Barbero, Cesar; universidad nacional de rio cuarto, quimica }\end{array}$ \\
\hline
\end{tabular}

\section{s ScholarONE \\ Manuscript Central}




\title{
Fabrication of Highly Ordered Arrays of Platinum
}

\section{Nanoparticles Using Direct Laser Interference Patterning}

\author{
Diego F. Acevedo ${ }^{a}$, Horacio J. Salavagione ${ }^{b}$, Andrés F. Lasagni $^{c}$, Emilia Morallón $^{b}$, Frank Mücklich $^{c}$ \\ and César Barbero ${ }^{a}$ \\ a Departamento de Química, Universidad Nacional de Río Cuarto \\ Ruta Nacional 36 Km 601, X5804ZAB. Río Cuarto. Argentina \\ ${ }^{\mathrm{b}}$ Departamento de Química Física e Instituto Universitario de Materiales, Universidad de Alicante, \\ Apartado 99, 03690 Alicante, Spain \\ ${ }^{\mathrm{c}}$ Department for Materials Science, Functional Materials, Saarland University, P.O. Box 1511 50, D - \\ 66041 Saarbruecken, Germany \\ E-mail: cbarbero@exa.unrc.edu.ar
}

RECEIVED DATE (to be automatically inserted after your manuscript is accepted if required according to the journal that you are submitting your paper to)

Corresponding Author: Dr. Cesar Alfredo Barbero

Departamento de Química, Universidad Nacional de Río Cuarto

Ruta Nacional 36 Km 601, X5804ZAB

TE: 00543584676157

FAX: 00543584676233

E-mail: cbarbero@exa.unrc.edu.ar 


\begin{abstract}
Highly ordered electrode arrays composed by lines of platinum nanoparticles deposited onto gold substrates have been made by direct laser interference patterning (DLIP) of polyaniline (PANI) thin films, followed by electrochemical deposition of platinum nanoparticles. Nanostructured arrays of electrocatalytic Pt particles are built in that way.
\end{abstract}

KEYWORDS (Word Style "BG_Keywords"). Nanoparticles array, interference patterning, polyaniline mask

BRIEFS. Highly ordered arrays of Pt nanoparticles DLIP of PANI

\title{
MANUSCRIPT TEXT
}

\section{Introduction}

The synthesis of ordered nanomaterials is one of the key points in the development of novel nanotechnologies. For several applications, the control of the distribution of the nanomaterials onto substrates is as essential as to precisely control their size. One particularly interesting market is that of power sources for portable applications using micro fuel cells (1). In those systems, a careful design could improve significantly the performance while the nature of the application allows for higher fabrication cost than in larger cells. Previous investigations of the construction of micro fuel cells can be grossly separated into two approaches: (i) a top-down approach involving miniaturizing existing designs for polymer-electrolyte-membrane fuel cells (2), and (ii) a bottom-up approach employing 
microfabrication techniques developed for the semiconductor industry to fabricate fuel cell structures (3). Here we describe the development and testing of highly ordered electrodes built of nano/micro lines of platinum nanoparticles electrodeposited onto gold substrates for its application in DMFC. To do that, we use a combination of bottom-up and top-down techniques, similar to lithographic methods. Lithographic patterning of polyaniline (PANI) has been achieved by using acid (4), or base photogenerators (5). We have produced PANI patterns by hydrolyzing N-Nitrosated polyaniline by chemical (6) and photochemical (7) routes. Here, we used PANI patterns to mask the electrodeposition of Pt nanoparticles into periodic arrays. Direct Laser Interference Patterning (DLIP) of PANI thin films is used to produce the PANI patterns. The technique has several advantages: (i) periodic patterns of features with a well defined long range order in the micro/nano scale can be fabricated in few seconds; (ii) areas ranging from $\mathrm{mm}^{2}$ to $\mathrm{cm}^{2}$ can be structured in each laser pulse; (iii) neither clean rooms nor especial conditions are necessary; (iv) the size and periodicity can be adjusted easily in each pulse allowing tailoring of the electrode to the application.

\section{Experimental}

Materials: Aniline (Merk) was distilled prior to polymerization using reduced pressure. The rest of reagents; hexachloroplatinic acid $\left(\mathrm{PtCl}_{6} \mathrm{H}_{2}\right)$, sodium sulphate $\left(\mathrm{Na}_{2} \mathrm{SO}_{4}\right), \mathrm{N}$-methyl-2-pyrrolidone $(\mathrm{NMP})$ and sodium hydroxide $(\mathrm{NaOH})$ were used as received from the manufacturer.

Synthesis of PANI onto gold electrodes. The electrochemical synthesis of polyaniline was performed onto the gold electrode by cycling ( 3 times) the work electrode between -0.20 and $+1.10 \mathrm{~V}$ vs. SCE at $50 \mathrm{mV} . \mathrm{s}^{-1}$ in a solution consisting of $0.1 \mathrm{M}$ aniline in $1 \mathrm{M} \mathrm{HCl}$, using $\mathrm{Pt}$ as counter electrode. The polymerization was completed by cycling ( 15 times) the electrode between -0.20 and $+0.65 \mathrm{~V}$ vs. SCE at $50 \mathrm{mV} . \mathrm{s}^{-1}$ in the same solution. The film was washed with $1 \mathrm{M} \mathrm{HCl}$ for $\sim 2 \mathrm{~min}$ to free it of aniline.

Laser Interference experiments: A Q-switched Nd:YAG (YAG: yttrium aluminum Garnat) laser (Spectra Physics) with a frequency of $10 \mathrm{~Hz}$ and pulse duration of $10 \mathrm{~ns}$ was used for the interference experiments. The laser beam was split into two sub-beams that were guided by mirrors to interfere on 
the sample surface. The period of the linelike pattern was $4.2 \mu \mathrm{m}$ and the utilized wavelength was 355 nm.

Platinum deposition. The platinum strips were electrodeposited in a 1 x $10^{-3} \mathrm{M} \mathrm{PtCl}_{6} \mathrm{H}_{2}+0.1 \mathrm{M}$ $\mathrm{Na}_{2} \mathrm{SO}_{4}$ electrolyte adjusting the $\mathrm{pH}$ at 11 by adding $\mathrm{NaOH}$. The potential was cycled between $0.0 \mathrm{~V}$ and $1.25 \mathrm{~V}$ at $100 \mathrm{mV} . \mathrm{s}^{-1}$ during 25 minutes (step 2).

Methanol oxidation. The electrochemical oxidation of methanol was carried out using a conventional cell of three electrodes using $0.5 \mathrm{M} \mathrm{H}_{2} \mathrm{SO}_{4}+0.1 \mathrm{M}$ methanol solution. The counter and reference electrodes were a platinum foil and a hydrogen reversible electrode immersed in the same working solution, respectively. Structured Pt nanoparticles array on Au and the Pt Nanoparticles onto flat Au were used as working electrode. The scan rate was $100 \mathrm{mV} . \mathrm{s}-1$ in all experiments.

\section{Results and discussions}

The sequential procedure to obtain the patterned electrodes is depicted in Scheme 1. First, a thin film of PANI is electropolymerized onto gold electrode. Very thin $(>10 \mathrm{~nm})$ and uniform films can be deposited in such a way (8). Then the PANI film was patterned using DLIP at ambient conditions, producing regular line arrays of PANI (Scheme 1, step 1) (9). Since the photothermal and thermal stability of Au and PANI are quite different it is possible to fabricate sets of regular lines of PANI and clean gold, separated by a fixed distance (period).

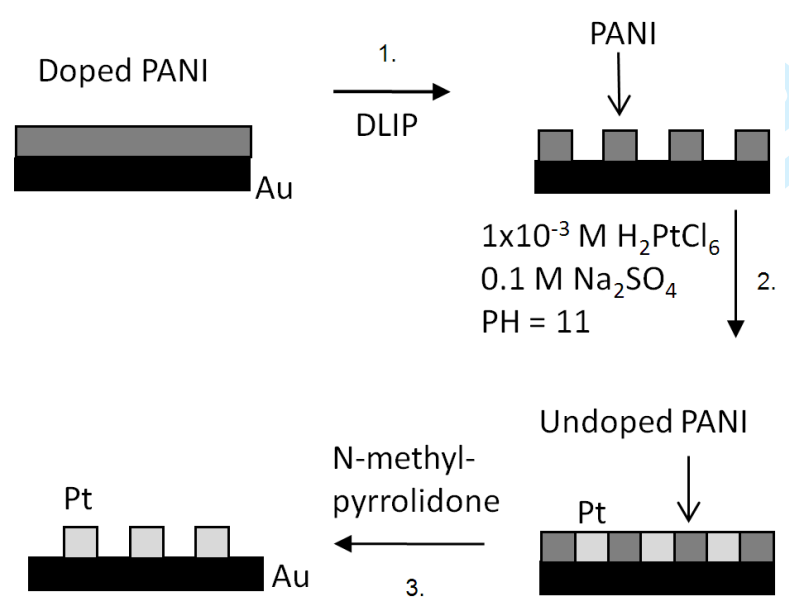


Taking into account that PANI behaves as an electrical insulator in neutral or alkaline solutions (10), we electrodeposited metals (in this work platinum) selectively onto the free gold lines (Scheme 1, step 2). The polymer mask allows deposition of the platinum only onto the ablated areas (see experimental for details). After that, PANI was removed using an appropriate solvent, N-methylpyrrolidone (NMP), leaving the electrode composed by alternated lines of platinum and gold (Scheme 1, step 3).
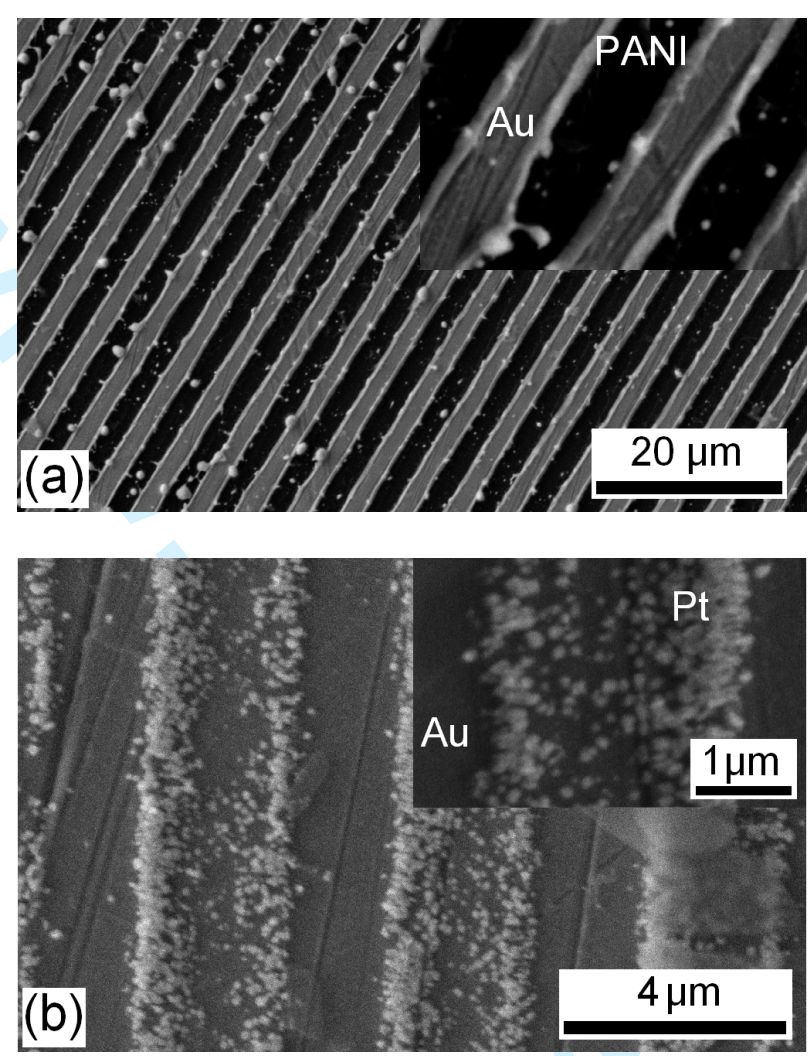

The topography of the electrode after the laser interference local ablation of PANI has been analyzed by White Light Interferometry (WLI). Well-defined arrays of polyaniline lines could be fabricated in one single laser pulse due to the local and periodic ablation of the polymer at the interference maxima positions (Fig S1a). Moreover, the width of the lines and their separation is very regular (Fig S1b). We measured a spatial period and height of $4.2 \mu \mathrm{m}$ and $347 \mathrm{~nm}$, respectively. Fig 1a shows the SEM image of the structure obtained after the ablation of the PANI film. The surface shows homogenous and ordered lines of polymer and gold of ca. 3 and $2 \mu \mathrm{m}$, respectively. In addition the micrographs reveal that the entire polymer was removed at the position where the light pattern has maximum intensity due to the local and periodical ablation. After that, platinum nanoparticles were deposited by electrochemical 
reduction of $\mathrm{PtCl}_{6}{ }^{-2}$ ions. Fig $1 \mathrm{~b}$ shows the SEM micrograph of the structure obtained after the electrodeposition of platinum and dissolution of the PANI with NMP. Quite regular lines of platinum nanoparticles that are electrodeposited only on the gold lines could be observed. The presence of nanoparticles is related to the metal nucleation in specific sites. The size of the lines of platinum particles build in this easy way has a width of ca. $2 \mu \mathrm{m}$, corresponding to the size of the free gold lines. The results show that arrays of Pt nanoparticles could be straightforwardly fabricated using DLIP of PANI to produce a mask. The method could be easily extended to other substrates e.g. platinum, glassy carbon, indium tin oxide (ITO). It also could be used to deposit nanoparticles of other active metals such as ruthenium, palladium or iridium.

Additionally, it has been shown that DLIP is able to ablate spots and other patterns, beside lines (11). Therefore, the structured deposit could be in the form of dot arrays or other periodic patterns.

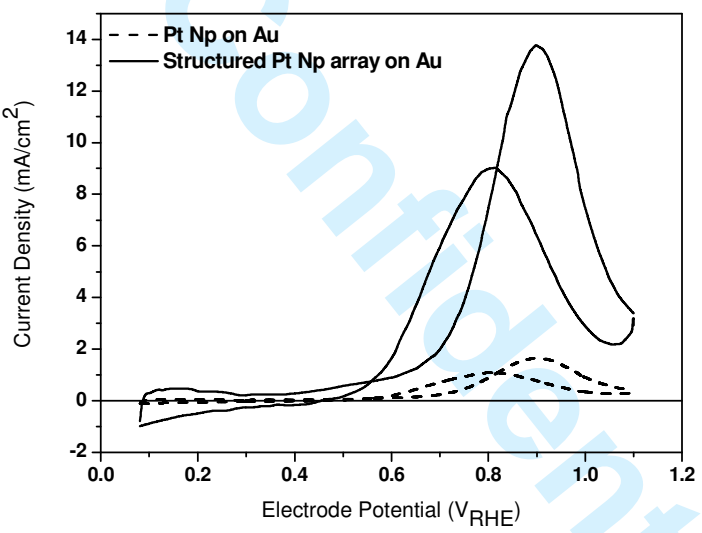

Finally, we tested the ability of the periodic arrays to act as electrodes. Since methanol is electrochemically oxidized on $\mathrm{Pt}$ and not on $\mathrm{Au}$ we used the reaction to detect the presence of Pt. The performance of the electrode for the methanol oxidation was evaluated using cyclic voltammetry (Fig 2). To assure that Pt nanoparticles has the same size, two areas, one flat and the other patterned, of the same Au electrodes were compared. The voltammograms display the typical behavior for methanol oxidation on Pt (Fig 2). However, the patterned array of nanoparticles shows a current density, calculated using only the area actually covered by particles, which is ca. 4.9 times larger than the one measured with and electrode made of nanoparticles deposited onto a non patterned surface. 
A possible explanation to the observed behavior is that the array of $2 \mu \mathrm{m}$ wide Pt nanoparticle strips on $\mathrm{Au}$ act as an array of ultra-microelectrodes (12). Therefore, the mass transfer of reactants and products to/from individual lines is cylindrical instead of planar as in a flat surface. Therefore, the reaction occurs at a faster rate on ultramicroelectrodes than on flat electrodes. Since methanol oxidation is controlled by the heterogeneous rate constant, it is unlikely that only increased diffusion of the reactant alters the current. On the other hand, it is known that poisoning of the Pt surface by $\mathrm{CO}$ and other side products of the oxidation controls the methanol oxidation rate (13). Therefore; an enhanced diffusion of those products could free the Pt surface increasing the methanol turnover on Pt.

We have demonstrated a robust method for the fabrication of highly ordered electrode arrays composed by strips of platinum nanoparticles deposited onto gold substrates. It is suggested that the patterned gold-platinum electrode acts as an array of ultra-microelectrodes. The method allows producing nanostructured patterns which could be useful in different research fields. Using the method is possible to fabricate structures made of electroactive oxides ( $\mathrm{NiOx}, \mathrm{CoOx})$ or even other conductive polymers such as polypyrrole, polythiophene or poly(3,4-ethylenedioxythiophene). In that way, nanostructured electrodes useful in batteries, supercapacitors could be easily built.

\section{ACKNOWLEDGMENT}

Alfred Krupp Foundation, Alexander von Humboldt Foundation, BMBF (Germany) FONCYT (PICT R03/453, PICT04/25521, PAE0422711), CONICET (PIP5221), SECYT-UNRC (Argentina) and MEC (MAT2007-60621) (Spain) are gratefully acknowledged. D.F.A and C.A.B. are permanent research fellows of CONICET

\section{SUPPORTING INFORMATION PARAGRAPH}

WLI images of a patterned PANI layer on gold using $126.75 \mathrm{~mJ} / \mathrm{cm} 2$ of laser fluence 


\section{FIGURE CAPTIONS}

Fig 1. Scanning Electron Micrographs of the electrodes. (a) PANI patterned by DLIP on Au (b) Pt nanoparticles array produced after Pt deposition on (a) and removal of the PANI lines.

Fig 2. Cyclic voltammograms of methanol (1 $\mathrm{M} \mathrm{CH} \mathrm{CH}_{3} \mathrm{OH}$ in $0.5 \mathrm{M} \mathrm{H}_{2} \mathrm{SO}_{4}$ ) oxidation on platinum particles deposited onto flat gold (dashed line); and platinum nanoparticles deposited on a patterned electrode (solid line). Scan rate $=100 \mathrm{mV} \cdot \mathrm{s}^{-1}$. The current density is calculated using only the area covered by Pt nanoparticles in each case.

\section{SCHEME TITLES}

Scheme 1. Fabrication procedure of the patterned gold-platinum electrodes: (1) Direct Laser Interference Patterning of PANI; (2) Electrochemical deposition of platinum; (3) Removal of the remnant PANI lines.

\section{REFERENCES}

(1) K. W Lux, K. J Rodriguez, Nano Lett. 2006, 6, 288-295

(2) B. H Liu, Z. P Li, K Arai, S. Suda, Electrochim. Acta. 2005, 50, 3719-3725

(3) S. Motokawa, M. Mohamedi, T. Momma, S. Shoji, T. Osaka, Electrochem. Commun. 2004, 6, $562-565$.

(4) M. Angelopoulos, J. M. Shaw, K. L. Lee, W. S. Huang, M. A. Lecorre, M. Tisier, J. Vac. Sci. Technol. B 1991, 6, 3428-3432. 
(5) G. Venugopal, X. Quan, G. E. Johnson, F. M. Houlihan, E Chin, O. Nalamasu., Chem. Mater. 1995, 7, 271-276

(6) H. J. Salavagione, M. C. Miras, C. Barbero, J. Am. Chem. Soc. 2003, 125, 5290-5291

(7) H. J. Salavagione, M. C. Miras, C. Barbero, Macromol. Rapid Commun. 2006, 27, 26-30

(8) C. Barbero, R. Kötz, J. Electrochem. Soc. 1994, 141, 859-865

(9) D. F. Acevedo, A. F. Lasagni, C. Barbero, F. Mücklich, Adv. Mater. 2007, 19, 1272-1275

(10) A. G. MacDiarmid, A. J. Epstein, Faraday Discuss. Chem. Soc. 1989, 88, 317-332.

(11) A. F. Lasagni, D. F. Acevedo, C. Barbero, F. Mücklich, Appl. Phys. A. Materials Science \& Processing. 2008, 91, 369-374

(12) I. Montenegro M. Arlete Queiros, J. L. Daschbach, Microelectrodes: Theory and Applications, Academic Publishers: Kluwer, 1991

(13) Y. Lin, X. Cui, C. Yen, C. M.Wai, J. Phys. Chem. B. 2005, 109, 14410-14415

\section{SYNOPSIS TOC}
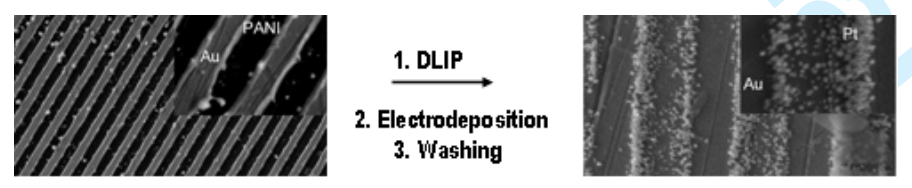

Highly ordered electrode arrays composed by lines of platinum nanoparticles deposited onto gold substrates have been made by direct laser interference patterning (DLIP) of polyaniline (PANI) films, followed by electrochemical deposition of Pt nanoparticles. 

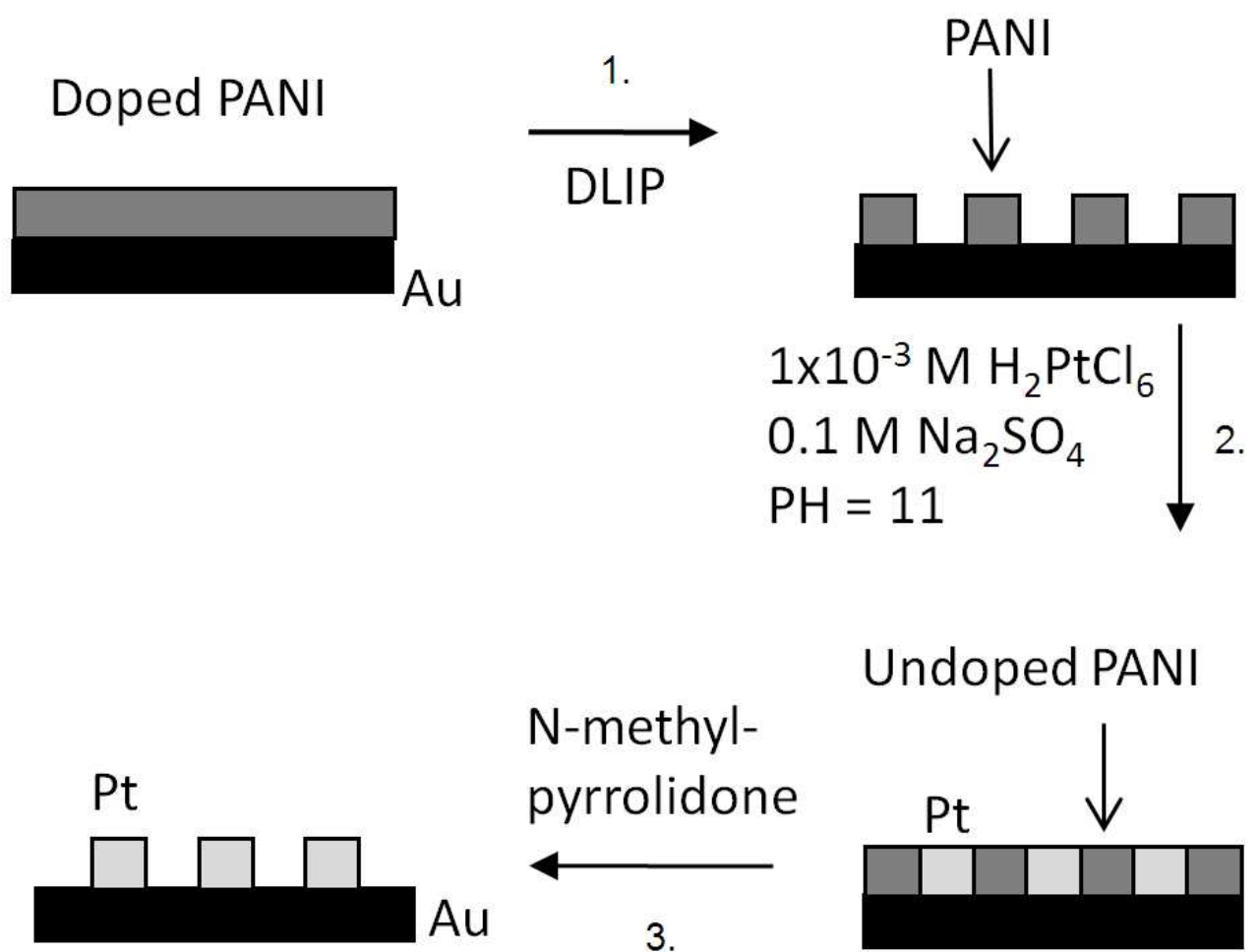

Undoped PANI

$252 \times 194 m m(96 \times 96$ DPI $)$

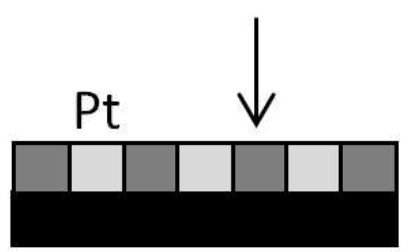




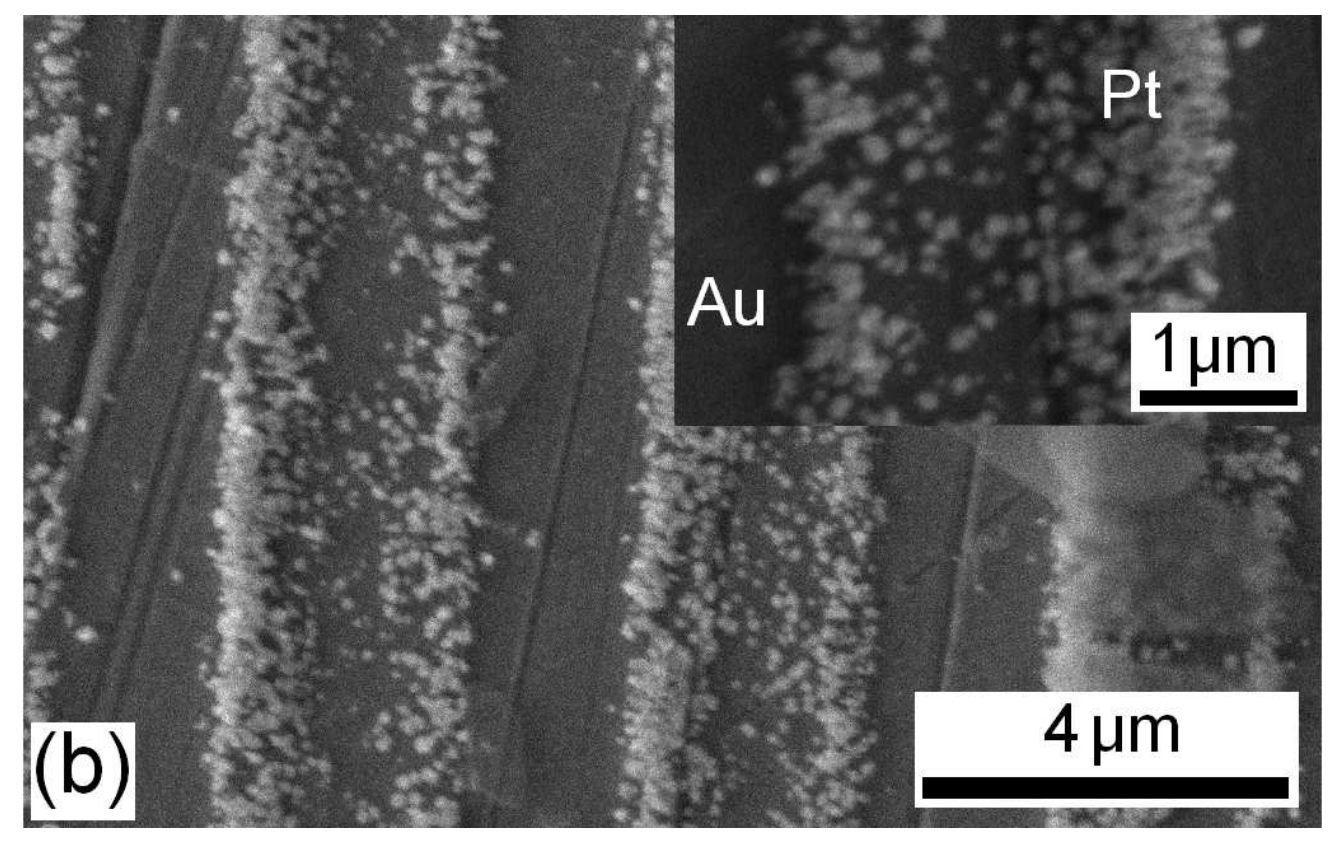

$270 \times 169 m m(96 \times 96$ DPI) 


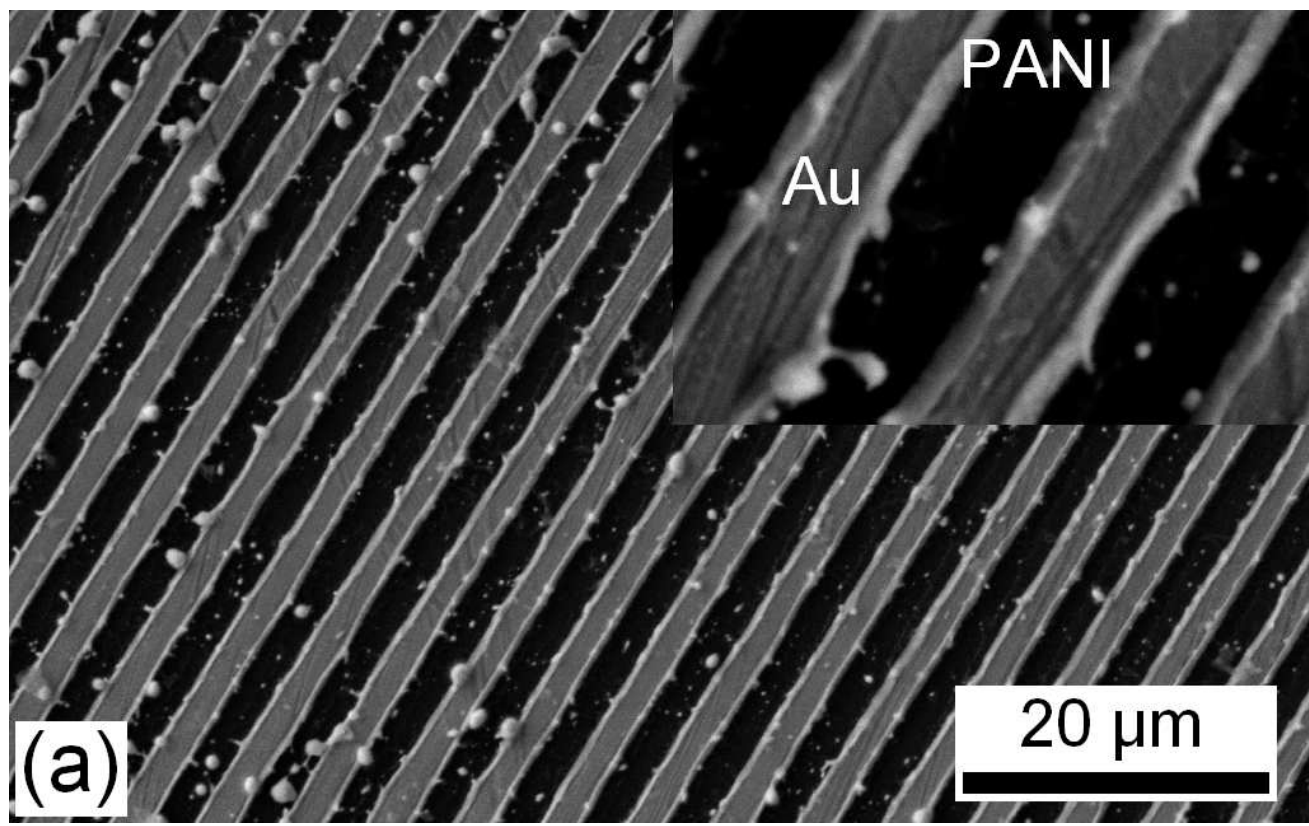

$270 \times 169 \mathrm{~mm}(96 \times 96$ DPI) 